\title{
Significados del consumo de sustancias psicoactivas en indígenas estudiantes de una universidad de Medellín, Colombia*
}

\author{
Meanings of Psychoactive Substance Consumption among \\ Indigenous Students from a University in Medellin, Colombia
}

Recibido: 10 de febrero de 2014 | Revisado: 30 de junio de 2015 | Aceptado: 30 de junio de 2015

\author{
ABRAHAM MENDOZA ** \\ JULIO FERNEY REYES ROMERO \\ GUILlERMO ANDRÉS GUTIÉRREZ MENDOZA \\ ISABEL CRISTINA POSADA *** \\ Universidad de Antioquia, Medellín, Colombia
}

doi:10.11144/Javeriana.upsy14-3.mpsc

Para citar este artículo: Mendoza, A., Reyes, J. F., Gutiérrez, G. A., \& Posada, I. C. (2015). Significados del consumo de sustancias psicoactivas en indígenas estudiantes de una universidad de Medellín, Colombia. Universitas Psychologica, 14(3), 975-984. http://dx.doi.org/10.11144/Javeriana.upsy14-3.mpsc

* Los autores declaramos que no existe conflicto de interés a nivel personal, comercial, académico, político o financiero. Agradecimientos: Al Comité para el Desarrollo de la Investigación (CODI), por su estrategia de sostenibilidad de 2013 a 2014 de la Universidad de Antioquia para el financiamiento de productos académicos.

** Administradores en salud. Correos electrónicos: amsaludp@gmail.com; guillermogmendoza@ gmail.com; julioreyrom@gmail.com

**** Psicóloga. Magíster en Salud Pública. Correo electrónico: isabel.posada@udea.edu.co
RESUMEN

Este artículo muestra el análisis de los significados del consumo de sustancias psicoactivas en indígenas estudiantes de la una institución universitaria de Medellín. Se utilizó la Teoría Fundamentada enraizada en el interaccionismo simbólico como técnica de análisis. Se realizaron seis entrevistas semiestructuradas y en profundidad y dos grupos focales, en 2012. Como resultados, se caracterizó la diferenciación de significados del consumo de plantas tradicionales y sustancias psicoactivas en comunidad indígena y occidental, y se identificó el papel que juega el contexto, la interacción con pares y la identidad cultural. Se concluye que a través de los conceptos que tienen los estudiantes indígenas sobre plantas tradicionales y sustancias psicoactivas, se evidencia una transformación de significados y prácticas de lo tradicional a lo occidental, resultante de conocimientos y vivencias adquiridas en la ciudad, interacción con el occidentalismo que puede resultar perjudicial a causa de la débil identidad cultural.

Palabras clave

sustancias psicoactivas; indígena universitario; identidad; plantas tradicionales; cosmogonía

\section{A B S T R A C T}

This paper shows the analysis of the meaning of psychoactive drug consumption among indigenous students from the university located in Medellín, Colombia, 2012. This study used grounded theory, rooted in symbolic interactionism as analysis techniques. Six semi-structured and in-depth interviews were conducted, and 2 focal groups. As a result, the authors characterized the difference in meaning that consuming traditional plants and psychoactive substances has for indigenous communities and for the western community. Likewise, they identified the role of context, peer interaction, and cultural identity. It is concluded that the students' concepts of traditional indigenous plants and psychoactive substances demonstrate that a transformation is taking place which westernizes the traditional meanings and practices. This is a consequence of the knowledge and experiences acquired in the city, an interaction with Occidentalism that can be detrimental, given their weak cultural identity.

Keywords

psychoactive substances; indigenous university student; identity; traditional plants; cosmogony 
Según la Organización Mundial de la Salud (OMS), la problemática del consumo de sustancias psicoactivas (SPA) es una de las principales preocupaciones en materia de salud pública a nivel mundial. La OMS señala que los trastornos mentales y neurológicos por abuso de sustancias son prevalentes en todas las regiones del mundo y son importantes factores que contribuyen a la morbilidad y a la mortalidad prematura (Gómez, Aical, Monllau, \& Vittore, 2010).

En Colombia, el último Estudio Nacional de Consumo de SPA en 2008 reveló que la prevalencia de uso de sustancias lícitas como el alcohol y el cigarrillo fue de $61.18 \%$ y de $21.46 \%$, respectivamente, para el año del estudio. También indicó que la proporción en el uso de sustancias ilícitas como la marihuana y la cocaína fue de $2.27 \%$ y de $0.72 \%$, respectivamente (Ministerio de la Protección Social \& Dirección Nacional de Estupefacientes, 2009). Este estudio además estimó que la ciudad de Medellín y su área metropolitana registran el mayor porcentaje de consumo de SPA, con una incidencia en el último mes de $41 \%$ de alcohol y de $20.86 \%$ de cigarrillo; al igual reveló que en esta ciudad el porcentaje de consumo de marihuana en el último año fue de 5.26 y de cocaína de 2.05 .

En la actualidad, el fenómeno relacionado con el consumo de SPA no ha dejado exentos los espacios universitarios, los cuales son utilizados como escenarios de oferta, demanda y proliferación de estas sustancias, ocupando un lugar significativo en la vida de los estudiantes. En el aprendizaje estudiantil cotidiano, propiciado por el vínculo con una compleja red social, converge con diversas prácticas e ideologías, evidenciándose así una asociación entre el inicio del consumo de SPA y los contextos sociales y culturales de la condición de universitario (Fundación Universitaria Luis Amigó [FUNLAM], 2009).

Según un estudio realizado en el 2008, en cuatro facultades de salud de la Universidad de Antioquia, titulado Consumo percibido en los pares y uso de drogas por los estudiantes universitarios: el estudio de caso Medellín/Colombia, el consumo de drogas está fuertemente relacionado con conductas de riesgo; la investigación demostró que los estudiantes universitarios tienen la mayor prevalencia de uso de drogas en las Américas, que la influencia de los pares es un factor clave asociado con el consumo y que existe una relación entre la percepción errónea del uso de drogas de los pares con el uso que hacen estudiantes universitarios (Montoya, Cunningham, Brands, Strike, \& Miotto Wright, 2009). En el 2009, la Red para la Investigación, la Formación y la Prevención del Consumo de Sustancias Psicoactivas en el Escenario Universitario (Red Unir), en su investigación, Del viaje en U: la vivencia universitaria y el consumo de sustancias psicoactivas, identificó que dentro de las drogas ilegales la marihuana sigue siendo la sustancia más representativa en la vivencia universitaria (FUNLAM, 2009).

El consumo de plantas tradicionales de las que se derivan las sustancias psicoactivas tienen un significado en los diversos pueblos indígenas. En Colombia, sin embargo, en la revisión de la literatura no se ha encontrado que alguna investigación se haya ocupado de los significados del consumo de estas plantas procesadas y catalogadas como SPA en indígenas universitarios. Por esta razón, se consideró necesario establecer cuál es el cambio que ocurre en las significaciones sobre SPA cuando esta población llega a vivir a una ciudad como Medellín, considerada como una de las ciudades con mayor prevalencia de consumo de SPA, lo cual posibilita un contexto diferente (Ministerio de la Protección Social \& Dirección Nacional de Estupefacientes, 2009).

Es por ello que el desarrollo de este estudio buscó involucrar a los estudiantes indígenas de la Universidad de Antioquia, indagando por sus significados sobre el consumo de sustancias psicoactivas. Los hallazgos servirían como soporte en la toma de decisiones para la implementación de programas de prevención y promoción por parte de la Universidad, beneficiando a la población indígena al reconocer su adaptación a nuevos contextos, la riqueza de su cosmovisión e identidad cultural y los posibles significados atribuidos al fenómeno del consumo de SPA.

\section{Metodología}

En el estudio de tipo cualitativo, se utilizó como herramienta metodológica la Teoría Fundamentada (TF), la cual se deriva de datos recopilados de manera sistemática y que son analizados por medio 
de un proceso de investigación. En este método, la recolección de datos, el análisis y la teoría que surgirá de ellos guardan estrecha relación entre sí. Asimismo, esta teoría parte del interaccionismo simbólico, que se refiere al "carácter peculiar y distintivo de la interacción entre personas. Esta peculiaridad consiste en el hecho de que las personas interpretan o definen las acciones de los otros en lugar de reaccionar ante ellas" (Coller, 2003 p. 245).

La población de indígenas matriculados en programas de pregrado de la Universidad de Antioquia para el año 2012 fue de $602^{1}$, los cuales procedían de diferentes regiones y etnias de Colombia. De ellos se eligió, por sus cualidades regionales y estudiantiles, una muestra representativa de 16 indígenas, con los que se logró la saturación de las categorías de análisis planteadas en la investigación. El empleo de la técnica del muestreo teórico, propia de la TF, se basa en la recolección de datos guiada por los conceptos derivados de la teoría que se está construyendo y en la noción de "hacer comparaciones". El propósito es acudir a lugares, personas o acontecimientos que maximicen las oportunidades de descubrir variaciones entre los conceptos y densifiquen las categorías en términos de sus propiedades y dimensiones (Strauss \& Corbin, 2002).

Las técnicas de recolección empleadas fueron la entrevista y los grupos focales. Las entrevistas, aplicadas a seis estudiantes, permitieron obtener información sobre una situación determinada, con el fin de lograr interpretaciones fidedignas del significado que para ellos tienen los fenómenos asociados a SPA, convirtiéndose así en una descripción del mundo vivido por los indígenas universitarios. Fueron dos los grupos focales, cada uno compuesto por cinco estudiantes, que desarrollaron una temática relativa a los conceptos, experiencias, emociones, creencias, temáticas, categorías o sucesos referidos

1 Comunidad o parcialidad indígena: es el grupo o conjunto de familias, de ascendencia amerindia, que tienen conciencia de identidad y comparten valores, rasgos, usos o costumbres de su cultura, así como forma de gobierno, gestión, control social o sistemas normativos propios que las distinguen de otras comunidades, tengan o no títulos de propiedad, o que no pueden acreditarlos legalmente o que sus resguardos fueron disueltos, divididos o declarados vacantes (Acuerdo Académico 236, 2002). al consumo de sustancias psicoactivas en estudiantes indígenas (Sampieri \& Fernández, 2007).

El plan de análisis en la TF de esta investigación consistió en dos procedimientos básicos: la codificación y la categorización, que se dieron durante todo el proceso, de la siguiente manera: aplicadas las primeras entrevistas para recolectar información, se procedió a codificarlas. Esta primera codificación recibe el nombre de codificación abierta, pues con ella se catalogó el mayor número posible de ideas aportadas por los entrevistados relacionadas con los objetivos de la investigación. Finalizada esta etapa, se procedió a generar unas categorías descriptivas, asíllamadas porque lo que se pretendió fue agrupar códigos articulados entre sí por el contenido de las ideas, pero sin hacer ningún análisis relacional de estos datos (Molina, 2009).

Las categorías descriptivas generadas indicaron al investigador cómo debía transformarse el instrumento para la recolección de la información de tal manera que ayudara a saturar estas categorías. Las entrevistas realizadas con este nuevo instrumento también fueron codificadas. Este procedimiento se conoce como codificación axial. Los nuevos códigos generados pasan a alimentar las categorías descriptivas con las que ya se contaba. Estos nuevos códigos vienen de grupos focales donde se rastrearon las posibles relaciones entre las categorías emergentes, es decir, que como se inició un proceso de análisis relacional de los datos, estas categorías pasan a llamarse categorías analíticas (Molina, 2009).

\section{Resultados y discusión}

A continuación, se presenta la categoría analítica central, que condensa la mayor cantidad de información aportada por los informantes, clave y de más relevancia para analizar los significados que dan los indígenas a las plantas tradicionales y sustancias occidentales $^{2}$. La categoría fue denominada: De lo sagrado al vicio: la transformación de significados, prácticas e identidades.

2 Para los indígenas el concepto "occidental" hace alusión a aquello que no pertenece a su propia comunidad, por ejemplo, la sustancia psicoactiva es un concepto elaborado por los occidentales. 


\section{De lo sagrado al vicio: la transformación de significados, prácticas e identidades}

El uso de sustancias psicoactivas, en la totalidad de los más antiguos grupos y tribus de cazadores y recolectores de distintos lugares del planeta, se remonta al comienzo mismo de la humanidad. Descubrimientos científicos revelan el empleo de algún tipo de sustancia psicoactiva acompañando cultos mágico-religiosos y actividades médico-terapéuticas. Cabe aclarar que, para muchos pueblos, medicina, magia y religión eran en un principio prácticas casi indisolubles; en ellas se utilizaban numerosas variedades de hierbas, hongos y plantas, ligadas a los procesos de comunicación espiritual, curación y prolongación de la vida y eran precedidas por autoridades indígenas o líderes considerados controladores de la naturaleza y cabezas médicas (Gutiérrez, 1983; Slapak \& Grigoravicius, 2006).

La autoridad espiritual hace uso de alucinógenos para lograr sus visiones, su fuente más importante de conocimiento. De esta forma esta se erige como una pieza fundamental en la convivencia de la cultura indígena, pues es el sacerdote o chamán quien, aparte de tener el poder de comunicación con los espíritus antepasados, también tiene la responsabilidad de educar a las futuras generaciones para la preservación de sus rituales, la identificación de los frutos sagrados de la madre tierra y las técnicas de consumo que soporten los procesos de sanación corporal y espiritual (Schultes \& Raffauf, 1994). Por ejemplo, la práctica del consumo de tabaco en comunidades indígenas es de uso medicinal y está restringida si se lleva a cabo individualmente; la única manera en que se puede consumir es con la presencia de la autoridad que en este caso son los médicos tradicionales, así lo hace saber un entrevistado de la etnia Pastos: "Tú no puedes como cualquier persona llegar allá y decir que como pertenezco al cabildo voy hacer una sanación utilizando esas sustancias o prácticas de uso particular que se dan solamente en los médicos tradicionales" (E2EPC17P4).

Aunque se sabe que los seres humanos han consumido sustancias psicoactivas desde hace miles de años sin que representara un problema para la so- ciedad, lo cual sí fue concebido como tal a fines del siglo XIX y principios del siglo XX. Esto puede explicarse por el hecho de que sociedades diferentes, con culturas disímiles, tienen distintas maneras de concebir la realidad, así como una misma sociedad transforma su manera de interpretar la realidad a consecuencia del devenir histórico y de los cambios políticos, sociales o económicos (Slapak \& Grigoravicius, 2006). En diversas culturas colombianas, la hoja de coca es un símbolo del mencionado poder, pues es medicinal, porque es natural y buena para el cuerpo y espíritu, a diferencia de la cocaína cuyos componentes químicos son perjudiciales para el organismo.

La hoja de coca, y lo que hoy es cocaína, son dos cosas muy diferentes, hoja de coca [...] la gran mayoría de indígenas en Colombia la utilizan como planta medicinal y una cosa muy diferente es la cocaína, que es el proceso que se realiza con una gran diversidad de ingredientes, [..] por ejemplo ya la cocaína lleva hasta ladrillo, [...] usted saca cocaína, pero el objetivo de sacar cocaína en usted es tener recursos económicos, isí o no?, usted no le importa al obtener recursos económicos no le importa el que se la vaya a consumir cual es el efecto que le va a producir mientras, que la hoja de coca, por ejemplo yo mambeo desde niño pero el efecto no es el mismo" [...]. (GF1ENC37)

Aunado a lo anterior, dada la ubicación de los asentamientos indígenas, se tiene una interacción perjudicial con grupos armados al margen de la ley o dedicados al narcotráfico. Tales grupos obligan agresivamente a las personas, tanto indígenas como campesinas, al sembrado de la hoja de coca para el lucro en la producción y venta. Un indígena de la etnia Inga expresa que el inicio del consumo de SPA en las comunidades indígenas se da por el narcotráfico, que impulsa a los integrantes de la comunidad a hacer parte de bandas y grupos armados.

Es que para nadie es secreto que en los diferentes contextos existe el narcotráfico de donde uno sea, existe narcotráfico, expender drogas, no solamente eso, conflictos internos, el cual viven solamente las 
partes regionales a nivel nacional, obviamente hay paisanos que ya prefieren dejar su comunidad y hacer parte de diferentes grupos, hasta incluso bandas. (GF1EI2C51)

Si bien es cierto que en las comunidades se consumen sustancias psicotrópicas, ya sea de índole alucinógena o embriagante, lo que se tiene en cuenta para determinar una construcción conceptual de planta sagrada o sustancia psicoactiva occidental es su significado en el consumo. Mientras que para las comunidades indígenas las plantas sagradas se consumen para tener un buen estado de salud y sobre todo por su implicación espiritual, la sociedad occidental consume SPA por placer y recreación.

El significado más en términos de sustancias psicoactivas en la ciudad para ese uso que se le da a las plantas medicinales con fines de vicio de pronto para sentirte bien y es algo que va a dañar tu salud, por eso es que en Occidente está prohibida, entonces por eso es que ponen letreros que dicen que no se pueden consumir. En cambio cuando se utilizan con fines medicinales te ayudan a sentir un bienestar general, y una armonía no solo en términos físicos sino en términos espirituales. (E2EPC62P11)

Por consiguiente, es inaceptable para las comunidades indígenas que se cataloguen las plantas sagradas como sustancias psicoactivas; sería una errónea clasificación, pues son sustancias esenciales en la percepción del mundo nativo y su significado trasciende el uso psicoactivo.

Según Mejía,

(...) el consumo de psicoactivos en el contexto mágico-religioso, no representaba un problema social ya que hacía parte de una cosmogonía, tales como el uso del vino, yagé y el cannabis, entre otros. Ahora bien, cuando todo este mundo de experiencias se desnaturalizan, estas sustancias que antes tenían nombres propios se transforman en "la droga", en un objeto mercantil, operación en la cual pierden su significado, e ingresan en la lógica del mercado. (Sánchez Upegui, 2002)
Sin duda alguna, con la llegada de los indígenas pertenecientes a diferentes etnias de todo el país a la ciudad, los significados sobre el consumo de plantas sagradas cambian. Manifiestan que en la ciudad es algo completamente diferente y que la forma de consumir es totalmente contraria a como se hace en las comunidades indígenas.

De esta manera, si bien las comunidades indígenas poseen creencias, hábitos y costumbres diferentes a los demás “occidentales", la llegada a la ciudad trae consigo múltiples cambios en su manera de ver el mundo, coadyuvando a que su identidad cultural $^{3}$ se pierda, por las costumbres o características que chocan con las que tenían arraigadas en su comunidad. En la población indígena sujeto de esta investigación, la identidad depende en gran medida de referentes "míticos", que se convierten en una percepción que termina siendo un sentimiento, en ocasiones una creencia. En el caso de los indígenas presentes en la ciudad y cascos urbanos municipales, se presenta como una mirada al pasado orientada a la recuperación de tradiciones, lenguas, historia, tejido social, saberes y haceres, los cuales son moldeados de modo activo en el proceso de construcción de identidades, relaciones sociales y prácticas económicas en la ciudad (Restrepo et al., 2004).

El carácter esencialista de lo "indígena" (atributos externos), valorado por la cultura hegemónica, adquiere para los indígenas un carácter instrumental usado para tener un lugar en la sociedad y va más allá del mero aspecto externo, pues representa la permanencia de un sentido implícito de identidad y autorreconocimiento por parte de un grupo diferente (Restrepo et al., 2004). Este argumento es confirmado por un estudiante indígena perteneciente a la etnia Nasa, quien expresa que el ser indígena y no dejarse permear por actitudes y comportamientos

3 En términos sociales, la identidad cultural es tanto una construcción individual como colectiva. Muchas colectividades representan una abstracción que no es más que el resultado de las relaciones humanas, de experiencias en común (costumbres, lengua, exclusiones sociales, política, etc.), no como una forma específica, sino formas de vida, maneras de vivir y de morir, un modo de pensar acerca de la razón de ser de la vida y de la muerte (Motta, 2006). 
occidentales es la identidad, es llevar los valores étnicos a todas partes: "Yo creo que ser indígena lo lleva aquí y en cualquier parte, y eso es identidad si, o sea el hecho de que usted sea siempre indígena usted va siendo indígena aquí y en toda parte" (GF1ENC62).

No obstante, cuando el indígena arriba a la ciudad, interacciona con un contexto donde la hibridación cultural presente muestra prácticas existentes que se van recombinando hasta constituir nuevas prácticas, en las que ritos del pasado son reordenados de acuerdo a una lógica diferente, dando como resultado manifestaciones de adaptación cultural, que buscan reducir el peligro representado en la globalización y la modernización, como una estrategia para asegurar la sobrevivencia de la cultura propia (Carvajal \& Van Horn, 2010).

Aunque en muchos casos esto se da, la identidad cultural puede ser permeada por todo tipo de transformaciones; cualquier persona que se encuentre provisional o definitivamente en otra cultura o sociedad, es vulnerable a todos los cambios que el medio ofrece y constreñido a aceptar elementos que favorecen el proceso de interrelación social (Carvajal \& Van Horn, 2010). Este proceso evidencia que la complejidad de la vida urbana debilita al indígena, permitiendo que el occidentalismo influya en sus comportamientos, consumos y preferencias, e incidiendo en que el indígena recién llegado a la ciudad se deje deslumbrar por la cultura "occidental" que le hace perder su arraigo cultural, tal y como lo argumenta un indígena de la etnia Nasa:

(...) hay un cierto cambio de identidad y (...) se genera cuando uno llega a estos contextos llamados ciudad, (...) entonces qué pasa, hay una debilidad, y esa debilidad permite también que el otro lo influya, y cuando lo influye obviamente lo va a permear y, bueno, si le queda gustando o sea es más de tipo familiar y de tipo cultural como decía hace rato, lo que pasa es que hay muchos indígenas que se creen blancos y que ya prefieren las leyes occidentales, y los mismos usos y costumbres, y eso sucede en los pueblos indígenas ya en todos, creo ya se ha permeado mucho. (GF1ENC63)
$\mathrm{Al}$ igual que en la ciudad, dentro del espacio universitario existe un sin número de redes constituyentes de identidad, debido a que es uno de los escenarios donde mayor interacción que facilita que estas se presenten. El indígena estudiante no es ajeno a esta dinámica, pues la mayoría del tiempo en la ciudad permanece en la universidad. Desde las primeras semanas de la vida universitaria, dentro y fuera de las clases, se van conformando pequeños grupos de amistades o intereses, entre indígenas y no indígenas (Restrepo et al., 2004). Es así como en la universidad existe diversidad de culturas, lo cual origina que se presente una construcción de nuevas identidades. Para sustentar este argumento un indígena estudiante de la etnia Zenú expresa que:

De pronto uno si busca tener compañía, tener amigos, pero es que la universidad se presta para que haya todo tipo de personas y de grupos o sea no solo vas a llegar a encontrar los drogadictos, los punkeros, los roqueros no, allá hay demasiada variedad de culturas de personas, de actividades. (GF2EZC77P12)

Alrededor del consumo de SPA en el campus universitario, se perciben factores de riesgo de tipo social, familiar, individual o psicológico; entre ellos, el de tipo social es uno de los más relevantes debido a que puede influir en cierta medida en el comportamiento de los miembros de la comunidad, por medio de procesos de comparación social, intercambio de información y en general por la socialización con otras personas (Krohn \& Thornberry, 1993).

Un estudio titulado: Consumo de drogas en jóvenes universitarios y su relación de riesgo y protección con los factores psicosociales, presenta como resultado que la ausencia o presencia de relación con personas consumidoras es el principal factor que actúa como protector o riesgo frente al consumo de todas las sustancias ilegales y legales (Cáceres, Salazar, Varela, \& Tovar, 2006). Este resultado es respaldado por las opiniones de un indígena estudiante entrevistado de la etnia Nasa que relata:

Yo siento que un compañero lo lleva a otro, porque, bueno yo cuando vivía sola yo no tenía ningún com- 
pañerito de esos y yo no hacía nada de esas cosas, pero una vez ya entre en contacto con cierto tipo de amigos que me invitaban a ciertas cosas y que decían no pero es que eso es normal, pues usted de donde viene, pues eso es muy normal acá, y uno como para no quedar mal, como para que no lo miren raro entonces uno como que empezaba a hacerlo, pero ya otras veces ya le gustaba demasiado (...). (E5ENC80P10)

Es importante considerar que los jóvenes universitarios se encuentran generalmente en ambientes sociales, y que una de las principales influencias sobre el consumo de SPA está relacionada con la actitud hacia estas por parte de las personas significativas, entre ellas, los amigos. De esta manera, los factores relacionados con la socialización y la influencia de los iguales afectarían el conocimiento, las creencias e intenciones respecto al consumo y, posteriormente, la decisión de consumir (Cáceres et al., 2006).

Este espacio universitario es facilitador de la interacción que se da entre jóvenes blancos y jóvenes indígenas, creando un vínculo social que afecta la subjetividad de cada individuo y que puede influir en la evolución de prácticas de consumo de SPA de manera problemática (Coller, 2003). Estas ideas se pueden cimentar en los relatos de los entrevistados que perciben que el consumo de SPA puede presentarse por el temor de ser excluido, y más para los indígenas que buscan aceptación social y acompañamiento por estar lejos de su lugar de procedencia. Un indígena de la etnia Pastos lo rememora de la siguiente manera: "Tú vas a una fiesta y todo el mundo va a consumir eso y por el temor de ser excluido de ese grupo pues vas a decir bueno yo voy a consumir" (E6EPC74P13).

Pero esta necesidad del indígena estudiante de identificarse con el otro, se fragmenta con la interacción de diversos aspectos occidentales, dejando atrás su propia identidad y acogiéndose a la construcción de identidades universitarias que se configuran a partir de colectivos basados en valores relacionados con estilos de vida y formas de ser, impuestos por el mercado y el consumo de todo tipo de mercancías, pues cuando se está en la universidad se asumen características y conductas propias de la vida en la ciudad (Coller, 2003).

Dichas conductas inciden propiamente en las acciones de occidentalización, que el indígena como catalizador y emisor de estas prácticas lleva a la comunidad, debilitando la propia identidad de los diversos pueblos indígenas y los amplios aspectos de diversidad cultural que poseen para la formación, el conocimiento y el consumo de plantas sagradas enfocadas a la curación, comunicación ancestral y demás rituales patrimoniales ejercidos con base en su cosmovisión y origen del mundo. Esto no solo debilita sino también propaga prácticas no inherentes al ser indígena. Lo anterior se puede inferir del desarrollo de esta investigación, especialmente de los relatos de los indígenas estudiantes que revelan que el consumo de SPA en indígenas estudiantes también afecta a la comunidad, pues posteriormente habrá una interacción entre el joven indígena y su comunidad, permeando conductas urbanas y occidentalizando así el consumo de SPA.

(...) Yo pienso que sí, más que todo por curiosidad, o porque de pronto hay muchachos que han salido a la ciudad y allá adquirieron como ese...pues tomaron esa costumbre (refiriéndose al consumo de SPA) no sé, y después vinieron acá (a la comunidad) y le dijeron a otros muchachos y así se va quedando. (E5ENC61P7).

La interacción indígena-occidental y viceversa que se evidencia en esta investigación demarca un fenómeno que por acciones de promoción no discriminantes se puede convertir en una situación problemática para los estudiantes indígenas y para sus comunidades. Es así como puede connotar diversas causas que inciden reciamente en el debilitamiento del arraigo cultural de cada estudiante foráneo nativo de las distintas etnias colombianas, trayendo como consecuencia la paulatina decadencia de las prácticas ancestrales, medicinales y tradicionales que dependen primordialmente de la formación familiar con miras al refuerzo de la identidad propia de cada comunidad. Esta situación seguramente se presenta en otras regiones de América, cuyos indí- 
genas migran a la ciudad en busca de oportunidades de formación.

\section{Conclusiones}

Los indígenas estudiantes consideran las plantas tradicionales como aquellas que tienen un significado sagrado, que sirven como medio de comunicación espiritual para curar las enfermedades de cualquier tipo que se presenten en la comunidad, pero encontraron en la ciudad que las sustancias psicoactivas son utilizadas con fines recreativos que llevan a un consumo problemático pues pueden ocasionar perjuicios.

Se identificó por medio de la investigación que la Universidad de Antioquia es vista por los indígenas estudiantes como un contexto determinante en el consumo de sustancias psicoactivas, y que la gran mayoría de indígenas estudiantes en su llegada a la ciudad buscan identificarse con un grupo afín, creando vínculos sociales que pueden llevar al olvido de sus raíces y su cultura.

La occidentalización en las tribus indígenas del país por medio de militarismo, medios de comunicación e indígenas universitarios origina rupturas en la fortaleza de la identidad cultural, fragmentando así los conceptos tradicionales que se tienen en la comunidad y deteriorando el conocimiento ancestral comunicado de generación en generación.

Se hace indispensable trabajar interdisciplinariamente con la participación de actores fundamentales no solo en el suministro de información, sino también en la formación de la riqueza cultural, integrada en una mirada compleja pero digna frente al contexto urbano en el que se vive, y propiciar campos de promoción de hábitos y prácticas saludables no solo en el ámbito universitario, sino también en el campo familiar y comunitario.

Se recomienda a las organizaciones indígenas promover e incentivar los estudios e investigaciones que generen conocimiento con base en las cosmovisiones de los pueblos indígenas, con el fin de fortalecer la identidad cultural de los indígenas estudiantes y sus comunidades.

\section{Referencias}

Acuerdo académico 236 de 2002 [Consejo Académico de la Universidad de Antioquia]. Por el cual se unifica el régimen de admisión para aspirantes nuevos a los programas de pregrado. 26 de octubre de 2002. Recuperado de http://secretariageneral. udea.edu.co/doc/u0236-2002.html

Cáceres, D., Salazar, I., Varela, M., \& Tovar, J. (2006). Consumo de drogas en jóvenes universitarios y su relación de riesgo y protección con los factores psicosociales. Universitas Psychologica, 5(3), 521-534.

Carvajal, O., \& Van Horn, D. (2010). El ser indígena en la ciudad: espacio de tensión y reelaboración de relaciones identitarias (Trabajo de grado de Antropología). Universidad de Antioquia, Medellín.

Coller, X. (2003). Canon sociológico. Madrid: Editorial Tecnos.

Fundación Universitaria Luis Amigó. (2009). Del viaje en $U$, la vivencia universitaria y el consumo de sustancias psicoactivas. Medellín: Departamento del fondo Editorial FUNLAM.

Gómez, R., Aical, C., Monllau, L., \& Vittore, G. (2010). Percepción de riesgo sobre las sustancias psicoactivas en jóvenes, padres y docentes del CBU de nivel medio de la ciudad de Córdoba: un estudio comparativo (Trabajo de grado para Licenciatura en Psicología). Universidad Nacional de Córdoba, Argentina.

Gutiérrez, V. (1983). Triple legado en la medicina tradicional colombiana. Bogotá: Editorial Presencia.

Krohn, M., \& Thornberry, T. (1993). Network theory: A model for understanding drug abuse among African-American and Hispanic youth. En M. R. De La Rosa \& J. -L. R. Adrados (Eds.), Drug abuse among minority youth: Advances in research and methodology (National Institute in Drug Abuse Research Monograph 130. NIH No. 93-3479), (pp. 102-128). Washington, DC: U.S. Government Printing Office.

Ministerio de la Protección Social \& Dirección Nacional de Estupefacientes. (2009). Estudio Nacional de Consumo de Sustancias Psicoactivas en Colombia, 2008. Bogotá: Editora Guadalupe.

Molina, D. (2009). Perspectiva de algunos actores sobre los modelos de educación para la salud subyacentes en programas de salud sexual y reproductiva dirigidos 
a adolescentes. Medellín 2004-2007 (Tesis de Maestría en Salud Pública). Universidad de Antioquia, Medellín.

Montoya, E., Cunningham, J., Brands, B., Strike, C., \& Miotto Wright, M. da G. (enero, 2009). Consumo percibido y uso de drogas lícitas e ilícitas en estudiantes universitarios en la ciudad de Medellín. Revista Latino-Americana de Enfermagen, 17(Supl. especial), 886-892. doi: 10.1590/S0104. 11692009000700020

Motta, N. (2006). Territorios e identidades. Revista Historia y Espacio, 26, 91-107. Recuperado de http:// historiayespacio.univalle.edu.co/TEXTOS/26/ Territorio\%20e\%20Identidades.PDF

Restrepo, Z., Bustamante, L., Collazos, C., Gaitán, M., Jacanamijoy, E., \& Motato, J. (2004). Voces indígenas universitarias: expectativas, vivencias y sueños. Reporte del proyecto de investigación: "situación del/ la estudiante indígena universitario/a, necesidades y perspectivas. Un estudio en Antioquia y Choco". Medellín: Organización Indígena de Antioquia y Universidad de Antioquia.

Sampieri, R., \& Fernández, C. (2007). Metodología de la investigación. México: McGraw Hill.

Sánchez Upegui, A. A. (2002). Del consumo ritual a la adicción contemporánea. Revista Universidad de San Buenaventura - Medellín, 16, 41-49.

Schultes, R., \& Raffauf, R. (1994). El bejuco del alma, los médicos tradicionales de la amazonia colombiana, sus plantas y sus rituales. Medellín: Universidad de Antioquia.

Slapak, S., \& Grigoravicius, M. (2006). Consumo de drogas: la construcción de un problema social. Anuario de Investigaciones, 14, 239-249.

Strauss, A., \& Corbin, J. (2002). Bases de la investigación cualitativa. Técnicas y procedimientos para desarrollar la teoría fundamentada. Medellín: Editorial Universidad de Antioquia. 
